\title{
Assessment of the feasibility of high-concentration capsaicin patches in the pain unit of a tertiary hospital for a population of mixed refractory peripheral neuropathic pain syndromes in Non-diabetic patients
}

\author{
Marc Giménez-Milà ${ }^{1 *+}$, Sebastián Videla ${ }^{2,3+}$, Marco-Antonio Navarro ${ }^{1}$, Adela Faulí ${ }^{1}$, Antonio Ojeda ${ }^{1}$, \\ Ana Bogdanovich', Luis-Alfonso Moreno', Clara Hernández-Cera ${ }^{1}$ and Carme Busquets ${ }^{1}$
}

\begin{abstract}
Background: High-concentration-capsaicin-patches (Qutenza $\left.{ }^{\oplus}\right)$ have been put on the market as a treatment for peripheral neuropathic pain. A minimum infrastructure and a determinate skill set for its application are required. Our aim was to assess the feasibility of treatment with high-concentration-capsaicin-patches in clinical practice in a variety of refractory peripheral neuropathic pain syndromes in non-diabetic patients.

Methods: Observational, prospective, single-center study of patients attended to in the Pain Unit of a tertiary hospital, $\geq 18$ year-old non-responders to multimodal analgesia of both genders. The feasibility for the application of capsaicin patch in clinical practice was evaluated by means of the number of patients controlled per day when this one was applied and by means of the times used for patch application.

Results: Between October 2010 and September 2011, 20 consecutive non-diabetic patients (7 males, 13 females) with different diagnoses of refractory peripheral neuropathic pain syndromes, with a median (range) age of 60 (33-88) years-old were treated with a single patch application. The median (range) number of patients monitored per day was not modified when the capsaicin patch was applied [27 (26-29)] in comparison with it was not applied [28 (26-30)]. The median (range) total time to determine and mark the painful area was 9 (6-15) minutes and of patch application was 60 (58-65) minutes. No important adverse reactions were observed.
\end{abstract}

Conclusion: High-concentration-capsaicin-patch treatment was feasible in our unit for the treatment of a population with refractory peripheral neuropathic pain. The routine of our unit was not affected by its use.

Keywords: Peripheral neuropathic pain, Capsaicin patch, Clinical practice

\section{Background}

Neuropathic pain management often involves the use of many therapeutic approaches (anti-epileptics, opioids, tricyclic antidepressants as well as noradrenaline and serotonin re-uptake inhibitors) that are not free of side effects [1-7]. In 2010, a new treatment (high-concentration

\footnotetext{
* Correspondence: magimene@clinic.ub.es

${ }^{\dagger}$ Equal contributors

'Pain Unit, Department of Anesthesiology, Hospital Clínic de Barcelona, Universitat de Barcelona, Carrer Villarroel 170, Barcelona 08036, Spain

Full list of author information is available at the end of the article
}

capsaicin patches, Qutenza ${ }^{\circ}$ ) for non-diabetic peripheral neuropathic pain in adults was put on the market. It was available in our hospital from October of that same year.

High-concentration capsaicin patches should be applied by a physician or by a health care professional under the supervision of a physician. Furthermore, specific skills and procedures should be applied according to the summary of the product [8]. They include as pretreating the area with a topical anesthetic prior to its application to reduce application related discomfort. This implies that solely applying the capsaicin patch requires 
an organizational infrastructure in the pain unit. In addition, within the context of the economic crisis, knowing whether this treatment can be conducted successfully in our facilities and what it implies may aid us in deciding if it should be included as an analgesic in our hospital. Therefore, the aim of this study was to assess the feasibility of the capsaicin patch applied in a pain unit of a tertiary hospital for peripheral neuropathic pain in nondiabetic patients who are non-responders to multimodal analgesia. Data on the tolerability, safety and effectiveness was also gathered.

\section{Methods}

\section{Study design}

This was an observational, prospective, single-center study of patients treated with the capsaicin patch in the Pain Unit. This study was based on the clinical practice without any modification. That is, the patients were administered the patches regardless of whether the study taking place. Therefore, this study simply took the opportunity to study their effects. This study was performed according to the stipulations of the Declaration of Helsinki and the level of protection of confidentiality concerning the protection of personal data as required by Spanish laws (LOPD 15/1999) was ensured. All patients gave their written informed consent for their medical information to be used for purposes of scientific research in accordance with the ethical committee of the participating site (Hospital Clinic de Barcelona). The Ethical Committee approved the informed consent in September 2010.

The capsaicin patch was applied following the instructions of the summary of product characteristics [8]. Moreover, blood pressure was measured with pressure cuffs (Critikon, GE Healthcare United kingdom, SA), every 10 minutes, from 15 minutes before applying the patch until 30 minutes after removing the patch and an electrocardiogram register was performed before and after patch application.

\section{Study population}

Patients included in this study had to fulfill the following eligibility criteria: men or women, $\geq 18$ years old with peripheral neuropathic pain, non-responders to multimodal analgesia and candidates to be treated with the capsaicin patch. No minimum VAS was required for inclusion. Patients with hypersensitivity to the active substance or to any of the excipients were not included.

The following data were gathered: age, sex, diagnosis, previous analgesic treatments, pain intensity, localization and area of pain, concomitant pain medication, indicators of feasibility, health state and adverse events related to the capsaicin patch as well as the effectiveness of the capsaicin patch.

\section{Feasibility for the application of capsaicin patch in clinical practice}

The indicators used to assess the feasibility in clinical practice were "number of patients visited per day when the patch was applied" and "number of patients visited the same day of the previous week if the patch was not applied in the Pain Unit". Likewise, as indicator of feasibility was used the times for patch application: "time to determine and mark the painful area", "time of patch application" and "time to monitor".

The "number of the patients visited per day when the patch was applied" was defined as the total number of patients visited in the Pain Unit the day that capsaicin patch was applied and corrected by the number of doctors who attended patients that day. The "number the patients visited the same day of the previous week if the patch was not applied in the Pain Unit" was defined as the number of patients attended to in the Pain Unit the same day of the previous week that the capsaicin patch was applied and corrected by the number of doctors who attended patients that day. The previous week was chosen whenever no patient was treated with capsaicin patch.

"Time to determine and mark the painful area" on the skin was defined as the total time (in minutes) needed by the physician to determine and mark the painful area. This variable includes: hair removal, washing and drying the skin, topical anaesthetic pretreatment and cutting the patch to match the size and shape of the treatment area. "Time of patch application" was defined as the total time (in minutes) from when the patch was applied until it was removed. "Time to monitor" was defined as the total time in minutes of monitored blood pressure from when the patient laid down on the couch until he/she was discharged from the unit.

\section{Tolerability and effectiveness}

The tolerability and safety of capsaicin patch (adverse events related to capsaicin patch application, electrocardiogram and blood pressure during treatment application) was gathered. Likewise, the pain intensity and its evolution (at 2 and 12 weeks after the treatment) was evaluated by means of a Numerical Rating Pain Scale (NRPS, ' 0 ' for no pain during the last 24 hours, and ' 10 ' for the worst possible pain during the last 24 hours) at baseline (before applying the capsaicin patch treatment) and 12 weeks later (last visit). Furthermore, the pain intensity was also gathered by means of a verbal NRPS through a telephone control 2 weeks after applying the capsaicin patch. Likewise, the neuropathic pain was diagnosed by the validated Spanish versions of the DN4 with a score equal to or above 4 and by the Brief Pain Inventory (BPI). The health state was measured by means of the validated Spanish version of the EuroQol- 
5D (descriptive and analogic) at baseline and 12 weeks later. Concomitant pain medications during the 12-week study were recorded.

\section{Statistical analysis}

No formal sample size was performed. The sample size was defined as the whole population treated with the capsaicin patch in the first year available in our hospital.

A descriptive analysis was performed to describe baseline population characteristics. Continuous variables were described as median (range: minimum-maximum) and categorical data were summarized as absolute frequency and percentages. As an exploratory analysis, the primary variable: the number of patients monitored per day in the Pain Unit when the capsaicin patch was or when was not applied was compared. The median and mean percentage changes in NPRS scores from baseline to 2 and 12 weeks later were calculated. The treatment success (responder) of the capsaicin patch was defined as a reduction of greater than $30 \%$ in pain intensity at 12 weeks from baseline. The percentage of responders and its corresponding 95\% confidence intervals ( $95 \% \mathrm{CI}$ ) were calculated. The correlation between pain intensity evolution and health state was assessed. Data was recorded and analyzed using Microsoft Excel running on Windows XP (Redmont, CA).

\section{Results}

\section{Patient characteristics}

A total of 20 consecutive patients ( 7 men and 13 women), who fulfilled the inclusion and exclusion criteria, were treated with the high-concentration capsaicin patch between October 2010 and September 2011 (both included) in its first year of availability in our hospital. All these patients were included in this study and all of them gave their consent. The median (range) age was 60 (33-88) years-old. The median (range) of the history of pain treated with the patch was of $4(0.6-14)$ years. The baseline characteristics of each patient included is shown in Table 1.

Prior to applying the capsaicin patch treatment, the diagnosis of peripheral neuropathic pain in non-diabetic patients was confirmed: post-herpetic neuralgia (6 patients), total knee arthroplasty post-surgical pain (3), painful scar (4: axilla, foot, shoulder and post-nephrectomy), femoral cutaneous neuropathy (2: in one patient with medical history of big hematoma in the leg), neuroma (3), complex regional pain syndrome (CRPS)-I after Colles' fracture (1) and HIV-associated neuropathy 1$)$. The median (range) DN4 score was 6 (4-10).

All patients included had been treated with multimodal analgesia (including interventional techniques in some cases). Previous multimodal analgesia is shown in Table 1. The concomitant analgesic treatments during the 12 week study were unchanged, that is, neither the doses nor the posology was increased.

\section{Feasibility for the application of capsaicin patch in clinical practice}

The capsaicin patch was applied to 20 consecutive patients without problems who were candidates for treatment (applicability 100\%).

The median number of patients monitored per day was not modified when the capsaicin patch was applied [27 (26-29)] in comparison with when the capsaicin patch was not applied [28 (26-30)]. The frequency for patients treated with the capsaicin patch was more or less every 2 weeks and only 2 patients were treated in the same week on only one occasion. Only in one case two patient were treated in two consecutive weeks and in the same day of the week, being the reference day the two same days of the previous week (two different weeks) when capsaicin was not applied.

The median (range) total time to determine, mark the painful area and apply local anesthetic was 9 [6-15] minutes. The median (range) total time of patch application was 60 (58-65) minutes. The median (range) time of blood pressure monitoring ("time to monitor") was 105 (99-109) minutes: 15 (14-18) minutes before applying the patch treatment and 29 (25-34) minutes for afterwards.

\section{Tolerability and effectiveness Tolerability and safety}

Pain during the capsaicin patch application (15 patients) was the most frequently reported adverse event. The pain intensity (NRPS) scored between 1 and 3. Only six (30\%) patients required the administration of oral NSAIDs. Erythema on the area where the capsaicin patch was applied was reported in 9 (45\%) patients, and ice was administered to $4(20 \%)$ patients to reduce the sensation of heat. Pruritus was reported in 1 (5\%) patient.

No alteration in the electrocardiogram register after capsaicin patch application was found. A slight increase in blood pressure (median of $4 \mathrm{mmHg}$ in mean arterial pressure) was observed in the first fifteen minutes of capsaicin patch application. This increment in blood pressure was neither statistically nor clinically significant.

\section{Effectiveness of the capsaicin patch}

All patients were pre-treated with a topical anesthetic cream (EMLA, Astrazeneca Farmaceutica Spain, S.A.) prior to application of a high-concentration capsaicin patch to reduce application related discomfort. The body areas treated were: thorax (7 patients), knee (3), thigh external side (2), leg external side (1), groin (1), foot (2), left flank (1), finger (2) and wrist (1).

The median (range) pain intensity at baseline was 8 (49) [mean (sd): 7.5 (1.7)] and it was 7 (0-9) [mean (sd): 5.8 (2.6)] at 12 weeks from the capsaicin patch treatment. The overall median (range) percentage of the reduction in pain 
Table 1 Baseline characteristics of all patients included $(n=20)$

\begin{tabular}{|c|c|c|c|c|}
\hline & Pain background (years) $^{a}$ & DN4 & Previous analgesic treatments & $\begin{array}{l}\text { Concomitant analgesic treatments } \\
\text { during } 12 \text { weeks study }\end{array}$ \\
\hline \multirow[t]{6}{*}{ Post-herpetic neuralgia } & 2 & 9 & $\begin{array}{l}\text { Opioids, } \mathrm{TL}^{\mathrm{b}}, \mathrm{NSAIDs} \text {, anticonvulsants, } \\
\text { antidepressants and } \mathrm{It}^{\mathrm{d}}\end{array}$ & $\begin{array}{l}\text { Opioids, TL, anticonvulsants } \\
\text { and antidepressants }\end{array}$ \\
\hline & 2 & 8 & $\begin{array}{l}\text { Opioids, TL, anticonvulsants, } \\
\text { antidepressants and IT }\end{array}$ & $\begin{array}{l}\text { Opioids, TL, anticonvulsants } \\
\text { and antidepressants, }\end{array}$ \\
\hline & 2 & 7 & $\begin{array}{l}\text { Anticonvulsants, Opioids, TL, } \\
\text { antidepressants and IT }\end{array}$ & Anticonvulsants \\
\hline & 24 & 4 & Anticonvulsants $\mathrm{TL}$, and IT & $\begin{array}{l}\text { Anticonvulsants, Opioids } \\
\text { and antidepressant }\end{array}$ \\
\hline & 5 & 8 & Opioids, $T L, L I^{c}$ and $I T$ & No treatment \\
\hline & 6 & 5 & Opioids, TL, NSAIDs, anticonvulsants and IT & Opioids and anticonvulsants \\
\hline \multirow{3}{*}{$\begin{array}{l}\text { Total knee arthroplasty } \\
\text { post-surgical pain }\end{array}$} & 4 & 6 & TL, NSAIDs, anticonvulsants and IT & Antidepressant and Opioids \\
\hline & 2 & 5 & Opioids, TL, anticonvulsants and IT & Opioids and antidepressants \\
\hline & 4 & 6 & LI' NSAIDs and IT & Opioids anticonvulsants and TL \\
\hline \multirow[t]{4}{*}{ Painful scar } & 0.6 & 6 & Opioids, TL, anticonvulsants and IT & Opioids, TL and anticonvulsants \\
\hline & 3 & 9 & TL and Opioids & TL and NSAIDs \\
\hline & 2 & 9 & Opioids, TL, NSAIDs and anticonvulsants & Opioids and anticonvulsants \\
\hline & 4 & 9 & Opioids, anticonvulsants and IT & Opioids, anticonvulsants, and TL \\
\hline \multirow[t]{2}{*}{ Femoral cutaneous neuropathy } & 14 & 9 & TL, NSAIDs and IT & $\mathrm{TL}$ \\
\hline & 6 & 9 & Opioids and anticonvulsants & Antidepressant \\
\hline \multirow[t]{3}{*}{ Neuroma } & 5 & 9 & $\mathrm{TL}$ and IT & No treatment \\
\hline & 5 & 9 & Anticonvulsants $\mathrm{TL}$, and IT & Anticonvulsants and NSAIDs \\
\hline & 10 & 9 & NSAIDs, anticonvulsants, IT, LI & No treatment \\
\hline CRPS-I & 1 & 9 & $\mathrm{TL}$ and IT & Anticonvulsants and antidepressants \\
\hline HIV-associated neuropathy & 5 & 9 & $\begin{array}{l}\text { Anticonvulsants Opioids, } \mathrm{TL} \text {, } \\
\text { and antidepressants }\end{array}$ & Anticonvulsants \\
\hline
\end{tabular}

CRPS-I: complex regional pain syndrome type I.

${ }^{\text {a }}$ Pain background: time (years) of pain related to the process studied.

${ }^{\mathrm{b}}$ Topical lidocaine.

'Lidocaine infusion.

${ }^{d}$ Interventional technique: neuroaxial blocks, intercostals blocks and sympathetic blocks.

scores after 12 weeks was $13 \%$ (-17\%, 100 [mean (sd): $23 \%$ (32)]. Eight (40\%, 95\% CI: 19\%-64\%) out of 20 treated patients were considered responders $(\geq 30 \%$ of reduction from baseline): 4 (57\%) out of 7 men and 4 (31\%) out of 13 women. In the group of responder patients, the median (range) percentage of the reduction in NPRS pain scores was 56\% (38\%-100\%) [mean (sd): $62.1(21-0)$ ], and in non-responders $11 \%(-17 \%, 25 \%)$ [mean (sd): 3.2 (13.6)]. Based on the responder definition of a pain reduction $\geq 50 \%$ from baseline, 5 (25\%, 95\% CI: 9\%-49\%) out of 20 patients treated with a high-concentration capsaicin patch [1 (14\%) man and 4 (31\%) women] were achieved a pain reduction $\geq 50 \%$ from baseline (Table 2 ). Figure 1 shows the percentage of responders for peripheral neuropathic pain diagnosis. It is worth noting that only 4 patients $(20 \%)$ did not previously receive an interventional treatment. Of those four patients, 2 patients were capsaicin patch responders. The evolution of pain is depicted in Figure 2.
Concomitant pain medications during the 12 weeks of study are summarized in Table 1 . No new medication was started during the 12 weeks of study. The patients carried on with the medication administered prior to capsaicin patch treatment because they presumably were on optimal doses.

The median (range) health state (EuroQol VAS) at baseline was 50 (20-90) [mean (sd): 50.8 (22.0)], and the descriptive system scored is shown in Table 1 . The health state at 12 weeks was 50 (20-100) [mean (sd): 55.3 (25.0)]. Six patients (30\%) had a EuroQoL VAS equal to or greater than 70, suggesting a good quality of life. In the group of responders, the EuroQol VAS at 12 weeks was a median (range) 80 (50-100) [mean (sd): 77.5 (19.2)] and it was 40 (20-80) [mean (sd): $44.2(20.2)$ ] in the no responder group; and the median (range) of change (baseline minus at 12 weeks) in the EuroQol VAS was of $12.5(-20,50)$ [mean (sd): 16.9 (23.7)] in responders and of $10[-70,30]$ in non-responders [mean (sd): 0.4 (27.3)]. 
Table 2 Health state evolution based on EuroQol-5D

\begin{tabular}{|c|c|c|c|c|}
\hline & \multicolumn{4}{|c|}{ Health state (EuroQol-5D) } \\
\hline & \multicolumn{2}{|l|}{ Baseline } & \multirow{2}{*}{$\begin{array}{l}\text { At } 12 \text { weeks } \\
\text { VAS }^{\mathrm{a}}\end{array}$} & \multirow{2}{*}{ Difference } \\
\hline & Descriptive & $\mathrm{VAS}^{\mathrm{a}}$ & & \\
\hline \multirow[t]{6}{*}{ Post-herpetic neuralgia } & 11121 & 60 & 75 & +15 \\
\hline & 22233 & 25 & 50 & +25 \\
\hline & 22232 & 50 & 40 & -10 \\
\hline & 11131 & 30 & 80 & +50 \\
\hline & 22232 & 25 & 40 & +15 \\
\hline & 12222 & 70 & 50 & -20 \\
\hline \multirow{3}{*}{$\begin{array}{l}\text { Total knee arthroplasty } \\
\text { post-surgical pain }\end{array}$} & 22232 & 90 & 20 & -70 \\
\hline & 21121 & 50 & 60 & +10 \\
\hline & 22333 & 30 & 40 & +10 \\
\hline \multirow[t]{4}{*}{ Painful scar } & 22333 & 20 & 30 & +10 \\
\hline & 11132 & 90 & 90 & 0 \\
\hline & 21333 & 35 & 20 & +15 \\
\hline & 22232 & 20 & 20 & 0 \\
\hline \multirow{2}{*}{$\begin{array}{l}\text { Femoral cutaneous } \\
\text { neuropathy }\end{array}$} & 11231 & 80 & 90 & +10 \\
\hline & 11232 & 50 & 80 & +30 \\
\hline \multirow[t]{3}{*}{ Neuroma } & 21222 & 70 & 80 & +10 \\
\hline & 12232 & 70 & 40 & -30 \\
\hline & 11231 & 50 & 100 & +50 \\
\hline CRPS-I & 11323 & 50 & 50 & 0 \\
\hline $\begin{array}{l}\text { HIV-associated } \\
\text { neuropathy }\end{array}$ & 21221 & 50 & 70 & +20 \\
\hline
\end{tabular}

${ }^{a}$ Visual Analogue Scale (VAS) of EuroQol-5D.

\section{Discussion}

This observational, prospective 12 -week study reports novel data on the feasibility (single 60-minute topical application) of the high-concentration capsaicin patch in patients who did not respond to multimodal analgesia. One of the most important reasons for performing this feasibility study was to identify both real and potential

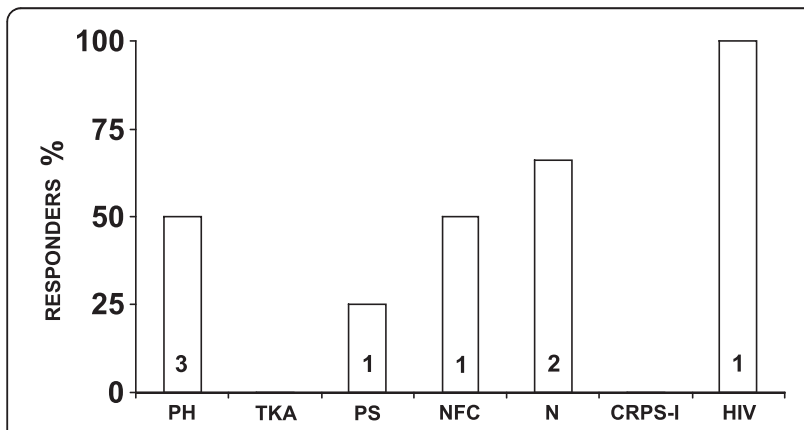

Figure 1 Responders to the capsaicin patch treatment by peripheral neuropathic pain diagnosis [PH: post-herpetic neuralgia (n: 6); TKA: total knee arthroplasty post-surgical pain (n: 3); PS: painful scar (n:4); NFC: Femoral cutaneous neuropathy (n: 2); N: neuroma (n: 3); CRPS-I: complex regional pain syndrome type I (n: 1); HIV: HIV-associated neuropathy (n: 1)]. problems that may be encountered in applying this new treatment (capsaicin patch) routinely in the clinical practice. Our pain unit has three treatment rooms, three consultations rooms, and we work altogether with two specialized pain nurses leading to a flexible work minimizing the negative impact of the treatment application. During the consultation clinic the physician could develop the normal activity because the nurses were trained in performing this procedure including the patient monitoring.

It is remarkable that no more than one patient was treated with a capsaicin patch per day and it was only in 1 week that the capsaicin patch treatment was applied to two patients. The aforementioned along with the small sample size may affect the interpretation of the feasibility data. However, the results suggest that the use of high-concentration capsaicin patch in our pain unit of a tertiary hospital may not have a relevant organizational impact insofar as the burden of visited patients was not affected the work-force. Thus, it can be carried out as planned within the estimated times.

The use of high-concentration capsaicin patches requires organization [8-10]. This study has allowed us, besides determining the potential impact that the treatment might have on our routine, to determine the adequacy of facilities and equipment that are presently available and how much real time is required to manage this treatment. The use of this treatment required a space where the treatment could be applied and the patient could be somewhat comfortable during the application. In addition, a nurse specialized in pain treatment is required for nearly 2 hours to apply the capsaicin patch, whose tasks are rescheduled to be performed during certain phases of the procedure, also a physician must be available at the facility.

In spite of the time required to manage this treatment, capsaicin patch application time did not affect the number of patients treated per day in our Pain Unit. The capsaicin patch was not a difficult treatment to manage and it might be an alternative in patients with peripheral neuropathic pain and multimodal analgesia non-responders.

Another objective of this feasibility study was to convince us of the treatment's value. Conclusions on the effectiveness of capsaicin patch could not be drawn due to the study design, the low number of patients included and to the variability of indications of peripheral neuropathic pain tested. To our knowledge, limited or no evidence based on clinical trials is available on the efficacy of the capsaicin patch for peripheral neuropathic pain different from post-herpetic neuralgia and HIV-associated neuropathy in non-diabetic patients $[11,12]$ or in diabetic polyneuropathy $[7,12]$. As expected, the group of patients diagnosed with post-herpetic neuralgia had a mean reduction of pain with respect to baseline similar to that reported in the registration clinical trials [13]. Likewise, if all patients treated with high-concentration capsaicin patch 

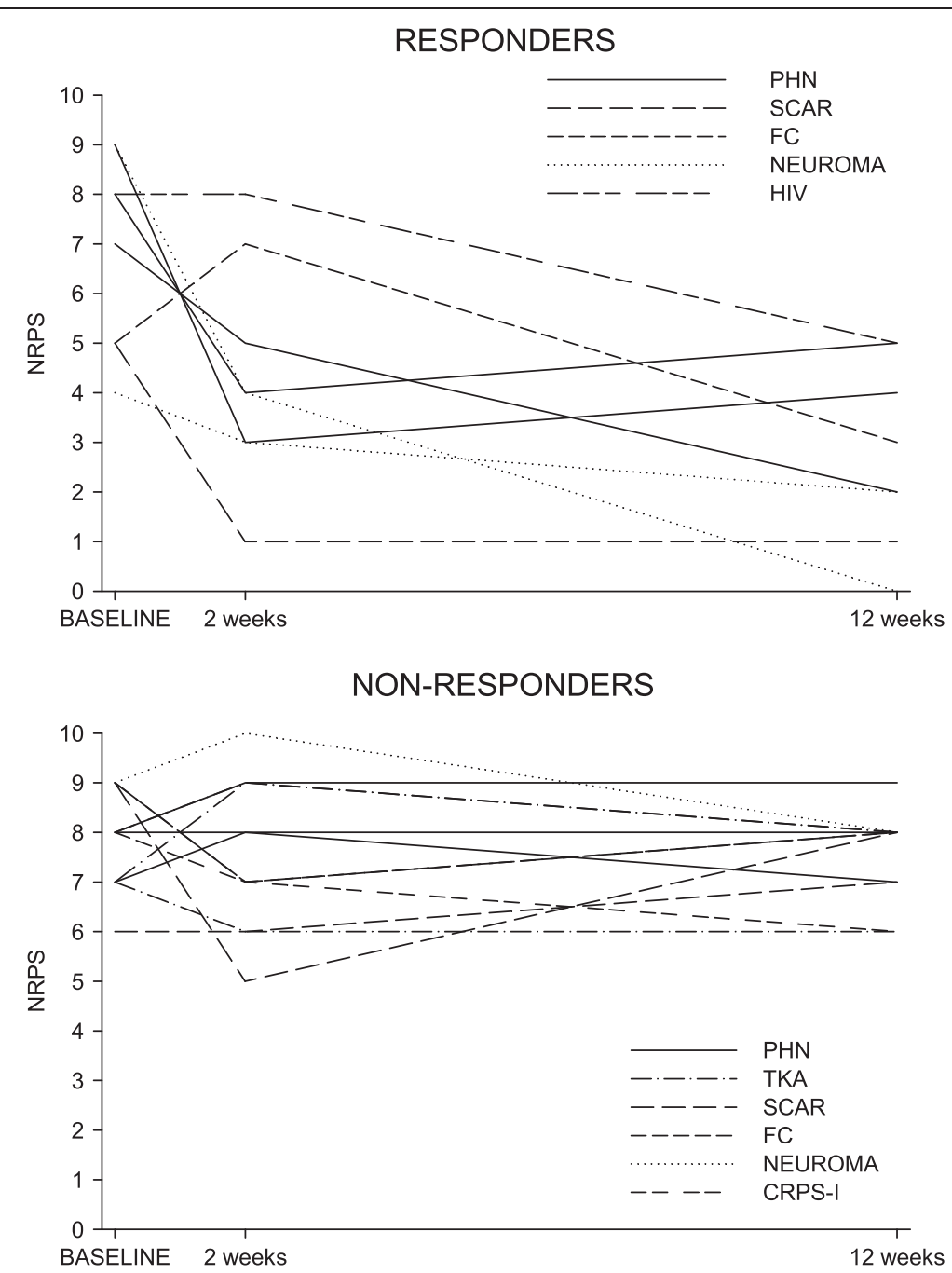

Figure 2 Evolution of pain according with responders and non-responders.

are considered, the overall mean pain reduction (23\%) was also the expected. It is remarkable that 6 responder patients had been treated with interventional techniques with no relief of pain before capsaicin patch. A possible explanation would be that the action of anesthetics on depolarization is limited in time. In contrast, capsaicin causes a defunctionalization which could last up weeks until recovery [14-16]. On the other hand, no interventional technique was performed on 2 responder patients, which represent $25 \%$ of responder patients. This could open a possibility for the capsaicin patch to be a therapeutic alternative in patients that have not received interventional treatment. Nevertheless, clinical trials of efficacy are required to answer this interesting question. Nonetheless, the reduction of pain seems to be associated with a health state improvement. The patients with a higher basal quality of life ( $>70$ VAS of EQ) are better responders. The descriptive analysis performed might suggest that the profile of responders might be related to the absence of problems with walking about, problems with self-care, no problems with the performance of usual activities and to a state of moderate anxiety or depression at the moment of capsaicin patch application. Likewise, the patients that responded to the capsaicin patch improved their quality of life.

The capsaicin patch was well tolerated. As expected, the most commonly reported adverse reactions were local transient pain at the application site, erythema and pruritus.

At this point, it is worth mentioning that in our patients topical anaesthetic pretreatment was only applied for a time less than 60 minutes as specified in the Summary of Product Characteristics. In no case was it necessary to remove the patch before time. To manage the acute pain during the application of the capsaicin patch, oral analgesics (NSAIDs) and local cooling (ice) were administered in $30 \%$ and $20 \%$ of the patients, respectively. In fact, shortacting opioids, as recommended in the summary of product characteristics, were not necessary. 
This study has limitations that should be considered before drawing any conclusion. The study design (observational and without control group) is not ideal for evaluating the impact of the capsaicin patch in clinical practice or for evaluating efficacy. The small sample size could lead to underestimating or overestimating the results obtained. In fact, this study was planned as exploratory. Data concerning the time of the analgesic treatment before inclusion in the study was not collected, and this might be affected the effectiveness. The evolution of pain was only based on 3 controls (at baseline, at 2 weeks and at 12 weeks). In this vein, when the study was planned, it was thought that the moment of pain intensity control (between 8:00 am to 3:00 pm) could not be standardized. In order to minimize the effect of variability at the moment of gathering the pain intensity, this one was based on the worst pain in the last 24 hours. Likewise, in our pain unit, the interventional procedures are performed twice a week in the operating theatre but on different days from capsaicin patch application. Hence, the feasibility results may not be applicable in hospitals with different organizational structure.

\section{Conclusions}

Data from this study provides evidence that the highconcentration capsaicin patch treatment was feasible in the pain unit of a tertiary hospital for the treatment of a variety of refractory peripheral neuropathic pain syndromes in a population of non-diabetic patients. The routine in our unit was not affected by its use. Nevertheless, pharmaco-economic studies are necessary to validate the feasibility of the capsaicin patch. No important adverse events were gathered.

\section{Competing interests}

S.V. has received a fee for collaborating with Laboratorios Dr Esteve in pain area. This work has not received funding. The authors declare that they have no competing interests

\section{Author's contributions}

MG-M, CB and SV designed and wrote the study protocol. MAN, AF, AO, AB and $L A M$ visited, monitored and followed up the patients, and applied the capsaicin patch. MG-M, CH-C and SV performed the statistical analysis and updated the database; all the authors participated in the analysis and interpretation of the data. MG-M, CH-C and SV drafted the manuscript, and all the authors reviewed it critically for important intellectual content. All the authors read and approved the final version of the manuscript.

\section{Acknowledgements}

Special thanks also go to the patients from Pain Unit and the nursing team M. Bellver and JL Gómez.

\section{Author details}

${ }^{1}$ Pain Unit, Department of Anesthesiology, Hospital Clínic de Barcelona, Universitat de Barcelona, Carrer Villarroel 170, Barcelona 08036, Spain. ${ }^{2}$ Catalan Society of Pain, Barcelona, Spain. ${ }^{3}$ Department of Experimental and Health Sciences. Faculty of Health and Life Sciences, Universitat Pompeu Fabra, Barcelona, Spain.
Received: 29 July 2013 Accepted: 11 December 2014

Published: 15 December 2014

\section{References}

1. Sindrup SH, Jensen TS: Efficacy of pharmacological treatments of neuropathic pain: An update and effect related to mechanism of drug action. Pain 1999, 83(3):389-400.

2. Jackson KC 2nd: Pharmacotherapy for neuropathic pain. Pain Pract 2006, $1: 27-33$

3. Cruccu G, Aziz TZ, Garcia-Larrea L, Hansson P, Jensen TS, Lefaucheur JP, Simpson BA, Taylor RS: EFNS guidelines on neurostimulation therapy for neuropathic pain. Eur J Neurol 2007, 14(9):952-970.

4. Dworkin RH, O'Connor AB, Backonja M, Farrar JT, Finnerup NB, Jensen TS, Kalso EA, Loeser JD, Miaskowski C, Nurmikko TJ, Portenoy RK, Rice AS, Stacey BR, Treede RD, Turk DC, Wallace MS: Pharmacologic management of neuropathic pain: Evidence-based recommendations. Pain 2007, 132(3):237-251.

5. Moulin DE, Clark AJ, Gilron I, Ware MA, Watson CP, Sessle BJ, Coderre T, Morley-Forster PK, Stinson J, Boulanger A, Peng P, Finley GA, Taenzer P, Squire P, Dion D, Cholkan A, Gilani A, Gordon A, Henry J, Jovey R, Lynch M, Mailis-Gagnon A, Panju A, Rollman GB, Velly A: Pharmacological management of chronic neuropathic pain - consensus statement and guidelines from the canadian pain society. Pain Res Manag 2007, 12(1):13-21.

6. Attal N, Cruccu G, Baron R, Haanpää M, Hansson P, Jensen TS, Nurmikko T: EFNS guidelines on the pharmacological treatment of neuropathic pain: 2010 revision. Eur J Neurol 2010, 17(9):1113-e88.

7. Anand P, Bley K: Topical capsaicin for pain management: Therapeutic potential and mechanisms of action of the new high-concentration capsaicin 8\% patch. Br J Anaesth 2011, 107(4):490-502.

8. Qutenza. Summary of Product Characteristics. http://www.ema.europa.eu/ docs/es_ES/document_library/EPAR_-_Product_Information/human/000909/ WC500040453.pdf [accessed 16.12.14]

9. Armstrong EP, Malone DC, McCarberg B, Panarites CJ, Pham SV: Costeffectiveness analysis of a new $8 \%$ capsaicin patch compared to existing therapies for postherpetic neuralgia. Curr Med Res Opin 2011, 27(5):939-950.

10. Darba J, Kaskens L, Perez-Alvarez N: Neuropathic pain: a budget impact analysis to estimate costs due to the introduction of Qutenza ${ }^{\circledR}$ on the Spanish market. Value Health 2011, 14:A411.

11. Clifford DB, Simpson DM, Brown S, Moyle G, Brew BJ, Conway B, Tobias JK, Vanhove GF: A randomized, double-blind, controlled study of NGX-4010, a capsaicin $8 \%$ dermal patch, for the treatment of painful HIV-associated distal sensory polyneuropathy. J Acquir Immune Defic Syndr 2012, 59(2):126-133

12. Webster LR, Peppin JF, Murphy FT, Lu B, Tobias JK, Vanhove GF: Efficacy, safety, and tolerability of NGX-4010, capsaicin 8\% patch, in an openlabel study of patients with peripheral neuropathic pain. Diabetes Res Clin Pract 2011, 93(2):187-197.

13. Qutenza, capsaicin. EPAR (European Public Assessment Report) summary for the public. http://www.ema.europa.eu/docs/en_GB/document_library/ EPAR__Summary_for_the_public/human/000909/WC500040448.pdf [accessed 16.12.14]

14. Backonja M, Wallace MS, Blonsky ER, Cutler BJ, Malan P Jr, Rauck R, Tobias J: NGX-4010, a high-concentration capsaicin patch, for the treatment of postherpetic neuralgia: A randomised, double-blind study. Lancet Neurol 2008, 7(12):1106-1112.

15. Nolano M, Simone DA, Wendelschafer-Crabb G, Johnson T, Hazen E, Kennedy WR: Topical capsaicin in humans: Parallel loss of epidermal nerve fibers and pain sensation. Pain 1999, 81(1-2):135-145.

16. Caterina MJ, Julius D: The vanilloid receptor: A molecular gateway to the pain pathway. Annu Rev Neurosci 2001, 24:487-517.

\section{doi:10.1186/1471-2253-14-120}

Cite this article as: Giménez-Milà et al:: Assessment of the feasibility of high-concentration capsaicin patches in the pain unit of a tertiary hospital for a population of mixed refractory peripheral neuropathic pain syndromes in Non-diabetic patients. BMC Anesthesiology 2014 14:120. 